\title{
Utilization of Non-Specific Staining to Visualise Liver Vasculature in Mice
}

Eva Szunyogova ${ }^{1,2}$ and Simon H Parson ${ }^{1^{*}}$

${ }^{1}$ University of Aberdeen, Institute of Medical Sciences, Aberdeen, United Kingdom

${ }^{2}$ Euan MacDonald Centre for Motor Neurone Disease Research, Edinburgh, United Kingdom

*Corresponding author: Simon H Parson, University of Aberdeen, Institute of Medical Sciences, Aberdeen, United Kingdom, Tel: 44 (0)1224 274328; E-mail: simon.parson@abdn.ac.uk

Rec date: Aug 02, 2015; Acc date: Aug 04, 2015 Pub date: Aug 06, 2015

Copyright: (c) 2015 Szunyogova E, et al. This is an open-access article distributed under the terms of the Creative Commons Attribution License, which permits unrestricted use, distribution, and reproduction in any medium, provided the original author and source are credited.

\section{Description}

Histological identification of morphological alterations are key indicators of functional defects. For example, liver disease which compromises perfusion and consequently function, is very often associated with abnormal tissue microvasculature. Traditionally an anti-platelet endothelial cell adhesion molecule-1 (PECAM-1/ CD31) antibody is utilized to visualize the vasculature, however in mouse liver this does not show the entire vasculature. The sinusoidal endothelial cells are a unique subtype of endothelial cells more closely structurally and functionally related to lymphatic vessels. Unlike other vessels, during postnatal development liver sinusoidal endothelial cells (LSECs) downregulate PECAM-1 levels to facilitate their high permeability [1]. Therefore to evaluate sinusoidal defects, another marker is required. Here we present a simple, effective and cheap method to visualise the entire liver vasculature utilization 'nonspecific' staining by an Fc antibody fragment (Figure 1).
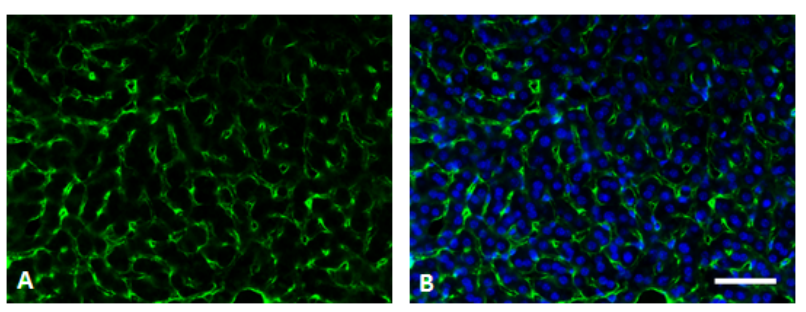

Figure 1: Fc fragment staining of mouse liver at postnatal day 20. A) Representative micrographs of $\mathrm{Fc}$ fragment staining of vasculature (green: left panel) and B) combined with DAPI stain for nuclei (blue: right panel). Scale bar $=50 \mu \mathrm{m}$.
Liver was harvested from mice sacrificed at postnatal day 20 (P20) and fixed for 4 hours in $4 \%$ paraformaldehyde $0.1 \mathrm{M}$ phosphate buffered saline (PBS). After fixation, the liver was washed in PBS and placed into $30 \%$ sucrose solution (made up in PBS) for subsequent OCT embedding. Liver sections $(5 \mu \mathrm{m})$ were air dried, washed in PBS and left in blocking solution ( $0.2 \%$ bovine serum albumin (BSA), $0.5 \%$ Triton X-100 (TX100) in PBS) for 2 hours. Once blocked, the slides were immunostained only with secondary antibody, Alexa 488goat anti-mouse IgG (Abcam, ab150113). All sections were visualised using a Nikon eclipse e400 microscope (20X objective) and its images captured using QICAM Fast 1394 camera and Improvision Velocity 4 image capture software.

The Fc fragment is most likely part of the IgG receptor expressed on LSECs that eliminates small immune complexes, thereby controlling complex-mediated autoimmune disease [2]. This simple process then visualises the entire liver vasculature in a single step.

\section{References}

1. Sugiyama Y, Takabe Y, Nakakura T, Tanaka S, Koike T, et al. (2010) Sinusoid development and morphogenesis may be stimulated by VEGFFlk-1 signaling during fetal mouse liver development. Dev Dyn 239: 386-397.

2. Ganesan LP, Kim J, Wu Y, Mohanty S, Phillips GS, et al. (2012) Fc $\gamma$ RIIb on liver sinusoidal endothelium clears small immune complexes. J Immunol 189: 4981-4988. 